\title{
Adaptive zooming in X-ray computed tomography
}

\author{
Andrei Dabravolski ${ }^{\mathrm{a}, *}$, Kees Joost Batenburg ${ }^{\mathrm{a}, \mathrm{b}}$ and Jan Sijbers ${ }^{\mathrm{a}}$ \\ ${ }^{a}$ iMinds-Vision Lab, University of Antwerp, Antwerpen, Belgium \\ ${ }^{\mathrm{b}}$ Centrum Wiskunde \& Informatica (CWI), Amsterdam, The Netherlands
}

Received 16 April 2013

Revised 11 September 2013

Accepted 4 November 2013

\begin{abstract}
.
BACKGROUND: In computed tomography (CT), the source-detector system commonly rotates around the object in a circular trajectory. Such a trajectory does not allow to exploit a detector fully when scanning elongated objects.

OBJECTIVE: Increase the spatial resolution of the reconstructed image by optimal zooming during scanning.

METHODS: A new approach is proposed, in which the full width of the detector is exploited for every projection angle. This approach is based on the use of prior information about the object's convex hull to move the source as close as possible to the object, while avoiding truncation of the projections.

RESULTS: Experiments show that the proposed approach can significantly improve reconstruction quality, producing reconstructions with smaller errors and revealing more details in the object.

CONCLUSIONS: The proposed approach can lead to more accurate reconstructions and increased spatial resolution in the object compared to the conventional circular trajectory.
\end{abstract}

Keywords: Acquisition geometry, adaptive zooming, computed tomography, prior information

\section{Introduction}

In most X-ray computed tomography (CT) acquisition setups, the source-detector system rotates around the object in a well-defined and geometrically simple manner. In micro-CT imaging, for example, a circular source-detector trajectory is by far the most popular one. The radius of such a trajectory is often chosen so as to avoid truncation in the acquired projections. That is, the radius is chosen large enough so that for each angle the full projection of the object is captured by the detector. However, for elongated objects, a circular trajectory does not allow to exploit the detector optimally. In [15], it was shown that non-planar trajectories yield visually better reconstructions than circular trajectories in applications of tomosynthesis to breast imaging. In single photon emission computed tomography (SPECT), non-circular orbits have been shown to reduce uniformity artefacts [14], to improve resolution [3,11], contrast, edge definition, and uniformity [5]. Nevertheless, the use of non-conventional trajectories is still almost unexplored.

\footnotetext{
${ }^{*}$ Corresponding author: Andrei Dabravolski, iMinds-Vision Lab, University of Antwerp, Universiteitsplein 1, 2610 Wilrijk, Belgium. Tel.: +32 32652840; Fax: +32 32652245; E-mail: andrei.dabravolski@uantwerpen.be.
} 
To improve reconstruction quality, a new approach is proposed in which the full width of the detector is exploited for every projection angle. To this end, projections are taken from the smallest possible distances to the object, while avoiding truncation. This is achieved by calculating the source position for every projection angle based on prior knowledge about the convex hull of the object. The proposed approach is integrated into an algebraic reconstruction framework. Possible applications of this approach include scanning devices with flexible acquisition geometries and mobile tomography devices. Objects with substantial differences in their dimensions, such as electronic components, can especially benefit from scanning based on the proposed approach.

Prior knowledge about the object can come in various forms. A total-variation (TV) minimization algorithm exploits sparsity of image derivative magnitude to address the few-view, limited-angle and bad-bin reconstruction problems [12]. In interior tomography, prior knowledge of the grey values within a small area inside the object is often readily available and can lead to more clinically feasible imaging [13]. In CT scanning protocols assuming repeated imaging, results of the initial scan(s) can be involved into the reconstruction of the consecutive scans allowing to significantly reduce the number of projections required [1]. Information about the edges of the object is shown to improve the reconstruction quality in the case of the few-view problem [4]. Finally, prior knowledge about the grey values of each of a few materials forming the object allows to use Discrete Algebraic Reconstruction Technique (DART), which can yield accurate reconstructions from a small number of projections or from a small angular range [2]. In all above-mentioned cases, prior knowledge is involved during the reconstruction. Our approach, on the contrary, uses the convex hull of the object as a source of information about the geometry of the object to optimise the acquisition. In practice, an approximation of the convex hull of the object can be built from a preparatory scan used to plan the scanning procedure or from CAD models (for industrial objects) [9].

The structure of this paper is as follows. In Section 2 our approach is explained. Section 3 describes experiment setups and presents reconstruction results. The approach is discussed in Section 4. Finally, conclusions are drawn in Section 5.

\section{Approach}

The idea of the proposed variable distance approach (VDA) is to acquire a projection for a particular projection angle by placing the $\mathrm{X}$-ray source as close as possible to the object, while avoiding truncation. In contrast to the circular trajectory approach (CTA), which keeps the source-object distance constant, VDA allows to fully use the detector and obtain more information from this angle. To calculate the smallest possible source-object distance, prior information about the object must be exploited. In our simulations, we use the convex hull of the object to calculate this distance.

Consider a cone-beam CT setup with a circular trajectory and a flat-panel detector (Fig. 1), where the source-detector distance is constant. Let $(x, y, z)$ be a Cartesian coordinate system in $\mathbb{R}^{3}$ which is fixed with respect to the object and let $O$ denote the centre of rotation. For a given projection angle, denote the source position on the circular trajectory with $S$ and the corresponding positions of the detector corners (in sequential order) with $D_{1}, D_{2}, D_{3}, D_{4}$. Suppose that the source-detector system can be shifted along the line $l$ containing $O$ and $S$.

Consider a point $P$ belonging to the pyramid $S D_{1} D_{2} D_{3} D_{4}$, which assures that the point $P$ is projected onto the detector. The source position closest to the point $P$ while avoiding truncation, say $S^{\prime}$, then corresponds to a case when $P$ belongs to one of the faces of the pyramid $S^{\prime} D_{1}^{\prime} D_{2}^{\prime} D_{3}^{\prime} D_{4}^{\prime}$ except for $D_{1}^{\prime} D_{2}^{\prime} D_{3}^{\prime} D_{4}^{\prime}$, where $S^{\prime} D_{1}^{\prime} D_{2}^{\prime} D_{3}^{\prime} D_{4}^{\prime}$ is obtained from $S D_{1} D_{2} D_{3} D_{4}$ by translation along $l$. Denote $s=$ 


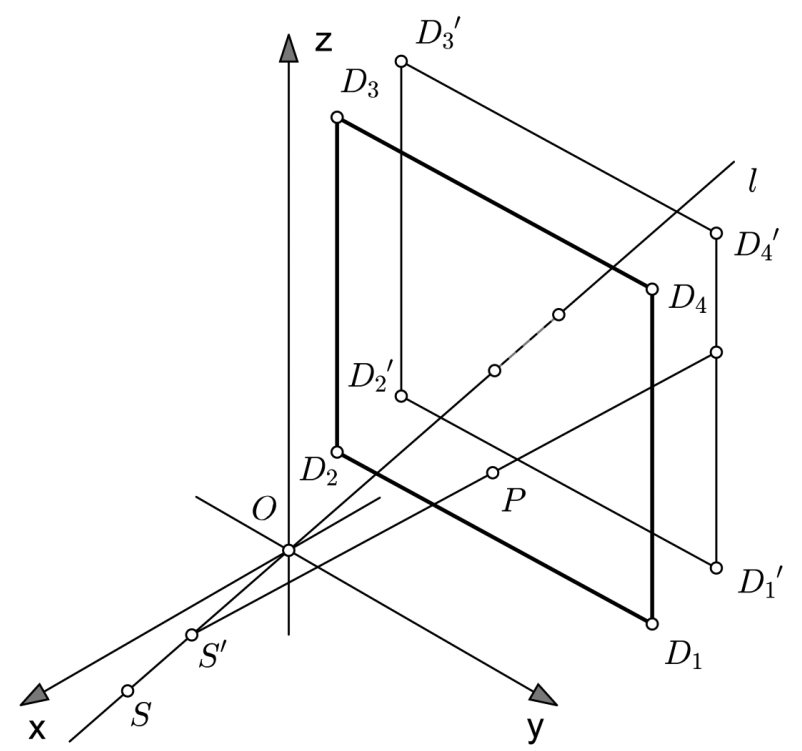

Fig. 1. Geometry of trajectory calculation in VDA.

$\overrightarrow{O S}, \boldsymbol{p}=\overrightarrow{O P}$ and the normal vectors of the faces $S D_{1} D_{2}, S D_{2} D_{3}, \ldots, S D_{4} D_{1}$ as $\boldsymbol{n}_{\mathbf{1}}, \boldsymbol{n}_{\mathbf{2}}, \ldots, \boldsymbol{n}_{\mathbf{4}}$, respectively. Assume that $P$ belongs to $S^{\prime} D_{i}^{\prime} D_{i+1}^{\prime}\left(1 \leqslant i \leqslant 4, D_{5}^{\prime} \equiv D_{1}^{\prime}\right.$ for ease of notation), which has $\boldsymbol{n}_{\boldsymbol{i}}$ as its normal vector. Then, the position vector $\boldsymbol{r}$ of any point in the plane containing $S^{\prime} D_{i}^{\prime} D_{i+1}^{\prime}$ (and $P$ ) can be found from

$$
\boldsymbol{n}_{\boldsymbol{i}} \cdot(\boldsymbol{r}-\boldsymbol{p})=0 .
$$

The intersection of $l$ and the plane defined by Eq. (1) is $s_{i, P}=t_{i, P} \boldsymbol{s}$, such that

$$
\boldsymbol{n}_{\boldsymbol{i}} \cdot\left(\boldsymbol{s}_{\boldsymbol{i}, \boldsymbol{P}}-\boldsymbol{p}\right)=0,
$$

which brings one to

$$
t_{i, P}=\frac{\boldsymbol{n}_{\boldsymbol{i}} \cdot \boldsymbol{p}}{\boldsymbol{n}_{\boldsymbol{i}} \cdot \boldsymbol{s}}
$$

$S^{\prime}$ can be found as $\overrightarrow{O S^{\prime}}=t_{P} s$, where

$$
t_{P}=\max _{1 \leqslant i \leqslant 4} t_{i, P}
$$

Consider $A_{1} A_{2} \ldots A_{n}(n \geqslant 4)$, the convex hull of the object. In our experiments, we suppose that the convex hull is a polyhedron, but the idea can be easily adapted to other cases. The closest possible source position $S^{\prime}$ for this convex hull can be expressed as $\overrightarrow{O S^{\prime}}=t s$, where

$$
t=\max _{P \in\left\{A_{1}, A_{2}, \ldots, A_{n}\right\}}=t_{P}
$$

From Eq. (5), the source position that is closest to the object while truncation is avoided can be computed. Repeating this procedure for every projection angle yields the desired trajectory. 
Table 1

MSE of the reconstructions of Phantoms 1-3 (shown in Fig. 2)

\begin{tabular}{ccc}
\hline & CTA & VDA \\
\hline Phantom 1 & & \\
$m=30$ & $9.10 \times 10^{-2}$ & $7.25 \times 10^{-2}$ \\
$m=200$ & $1.46 \times 10^{-2}$ & $1.35 \times 10^{-2}$ \\
Phantom 2 & & \\
$m=30$ & $7.66 \times 10^{-2}$ & $7.47 \times 10^{-2}$ \\
$m=200$ & $1.34 \times 10^{-2}$ & $1.31 \times 10^{-2}$ \\
Phantom 3 & & \\
$m=30$ & $1.58 \times 10^{-3}$ & $1.48 \times 10^{-3}$ \\
$m=200$ & $3.45 \times 10^{-4}$ & $3.47 \times 10^{-4}$ \\
\hline
\end{tabular}

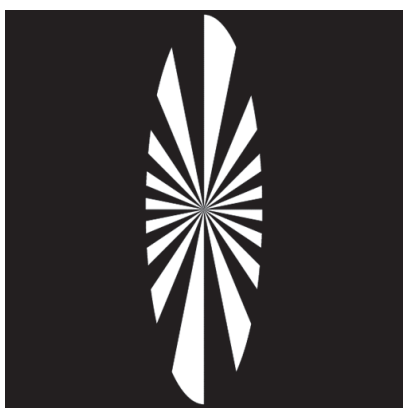

(a)

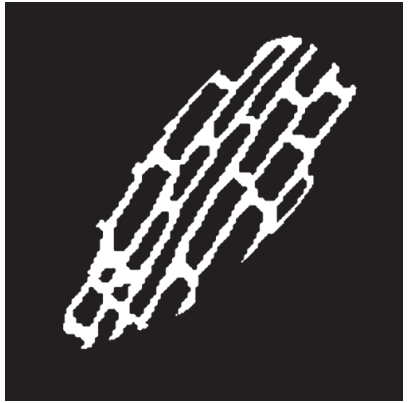

(c)

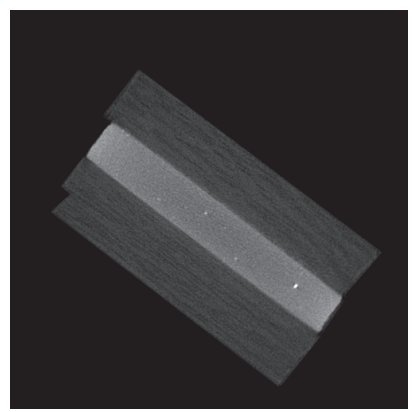

(e)

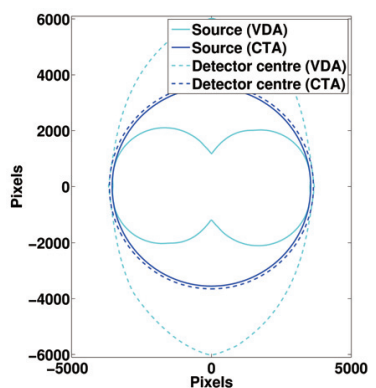

(b)

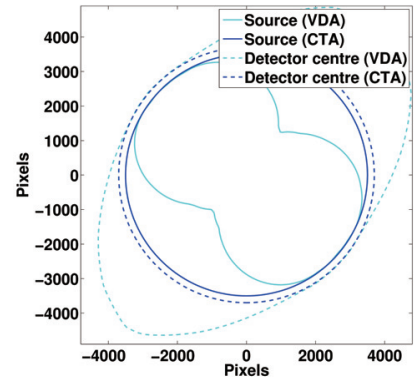

(d)

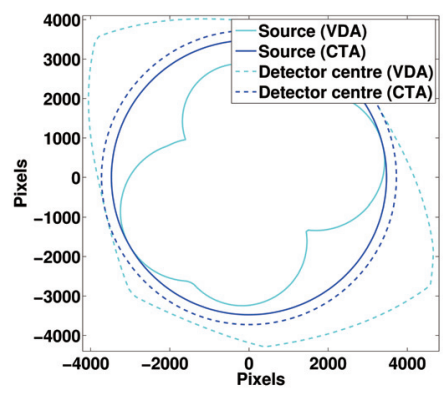

(f)

Fig. 2. Phantoms 1-3, $2048 \times 2048$ pixels (a, c, e), and the corresponding trajectories (b, d, f). (Colours are visible in the online version of the article; http://dx.doi.org/10.3233/XST-130410)

\section{Experiments}

\subsection{Noiseless simulations in two dimensions}

Simulation experiments were run using three phantom images (Fig. 2) to demonstrate the proposed approach. Phantom 1 (Fig. 2(a)) is a Siemens star-like phantom. Phantoms 2 and 3 (Figs 2(c) and 2(e)) 


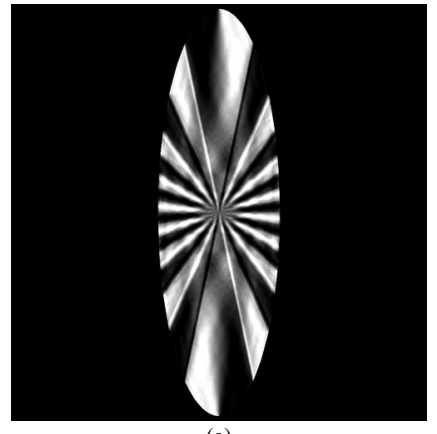

(a)

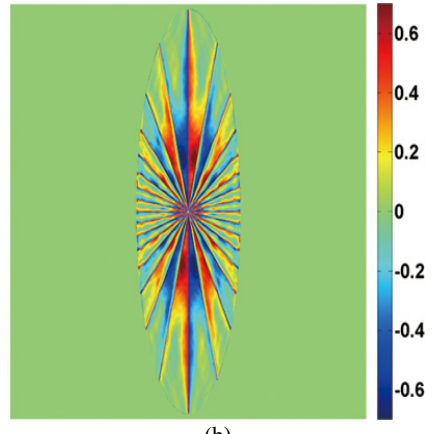

(b)

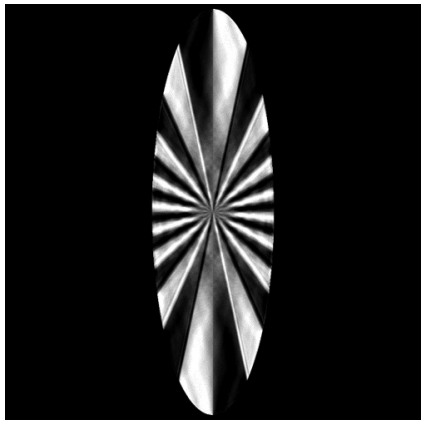

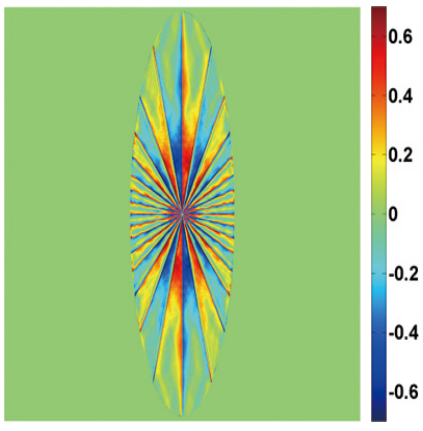

(d)

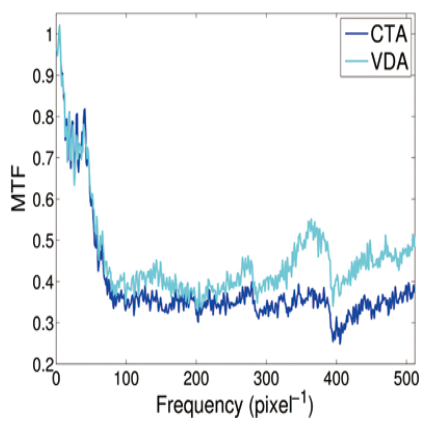

(e)

Fig. 3. Reconstructions of Phantom 1 (Fig. 2(a)) for CTA (a) and VDA (b), corresponding error images (c, d) and the modulation transfer function (e), $m=30$. (Colours are visible in the online version of the article; http://dx.doi.org/10.3233/XST-130410)

represent a fragment of foam and a fragment of pencil CT image, respectively. Reconstructions were performed on a square reconstruction grid of $1024 \times 1024$ pixels while the size of the each phantom was $2048 \times 2048$ pixels to reduce the effect of the pixelation on the reconstructions. A number of $m$ equiangular fan-beam projections were computed from the original phantoms using Joseph's projection method [8]. The source trajectory for VDA was calculated according to Eq. (5). It is shown in Fig. 2 together with the source trajectory for CTA and the detector centre trajectories. In CTA the source was placed at the distance corresponding to the maximum distance used in VDA. A detector with $n=$ 1024 elements was used. The reconstructions were built with 300 iterations of the Simultaneous Iterative Reconstruction Technique (SIRT) [6]. Values outside the convex hull were not involved in the reconstruction. All experiments presented in the paper were implemented using the ASTRA toolbox [10].

The quality of the reconstructions was assessed by calculating the mean squared errors (MSEs) according to

$$
\operatorname{MSE}(\tilde{I}, I)=\frac{1}{|C|} \sum_{(i, j) \in C}(\tilde{I}(i, j)-I(i, j))^{2},
$$

where $\tilde{I}$ denotes the reconstruction upsampled by splitting each pixel into $2 \times 2$ pixels and $I$ is the original phantom with the convex hull $C$. Table 1 shows the obtained numerical results. Fig. 3 shows the examples of reconstructions of Phantom 1 using CTA and VDA. These reconstructions suggest that VDA can yield visually better reconstructions, providing clearer feature borders, e.g. for vertical ray-like parts of the phantom (Figs 3(b) and 3(d)). From Table 1, it is clear that VDA is only slightly outperformed by 


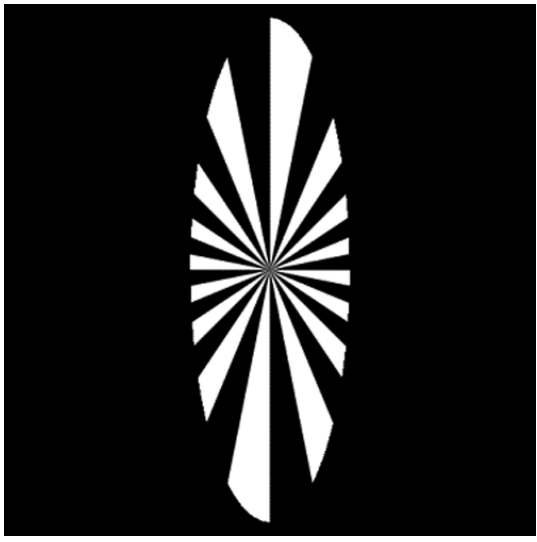

(a)

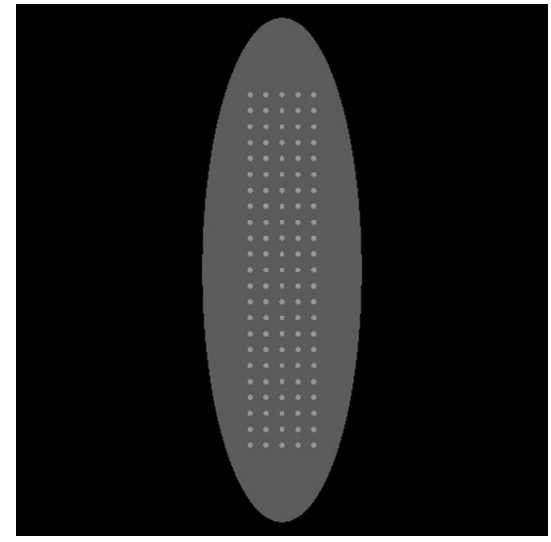

(b)

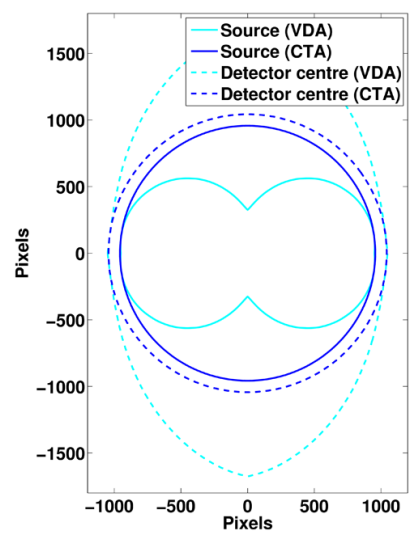

(c)

Fig. 4. Cross sections of Phantoms 4 (a) and 5 (b, windowed to [0.45, 0.55] for better visual contrast), $512 \times 512 \times 512$ pixels, by the plane $z=0.5$, and the trajectories used in reconstruction (c) (in the plane $z=0$ ). (Colours are visible in the online version of the article; http://dx.doi.org/10.3233/XST-130410)

CTA when reconstructing Phantom 3 from 200 projections, providing notably better figures for MSE in the remaining cases.

To further compare the proposed approach with its conventional counterpart, the modulation transfer functions (MTFs) of CTA and VDA were calculated as follows. First, the two-dimensional discrete Fourier transforms of the phantom and the reconstructions obtained using CTA and VDA were computed. Next, the magnitudes of the Fourier coefficients for the reconstructions were divided by the corresponding magnitudes of phantom's Fourier coefficients. Finally, the results were integrated for each frequency. Figure 3(e) presents the resulting MTFs for Phantom 1, which confirm the ability of the proposed approach to produce reconstructions with improved spatial resolution compared to CTA.

\subsection{Noiseless simulations in three dimensions}

Experiments were performed using two phantoms (Fig. 4). Phantom 4 (Fig. 4(a)) is in fact low resolution version of Phantom 1 (Fig. 2(a)) stacked 512 times and intersected with an ellipsoid having axial ratios 0.95:0.3:0.3. Phantom 5 (Fig. 4(b)) represents the same ellipsoid with a lattice-like structure consisting of voxel representations of spheres inside. Reconstructions were performed on a cubic reconstruction grid of $256 \times 256 \times 256$ voxels while the size of each phantom was $512 \times 512 \times 512$ voxels. A number of $m$ equiangular cone-beam projections were computed from the original phantoms using Joseph's projection method [8]. The source trajectory for VDA (Fig. 4(c)) was calculated according to Eq. (5). In CTA the source was again placed at the distance corresponding to the maximum distance used in VDA. The detector had $256 \times 256$ elements. The reconstructions were built with 300 iterations of SIRT. Values outside the convex hull were not involved in the reconstruction.

The quality of the reconstructions was assessed by a three-dimensional analogue of Eq. (6). Figures 5 and 6 present the resulting reconstructions. Figures 5(d) and 6(d) present the difference

$$
D\left(\tilde{I}_{C T A}, \tilde{I}_{V D A}, I\right)=\left|\tilde{I}_{C T A}-I\right|-\left|\tilde{I}_{V D A}-I\right|
$$

showing, which approach produces the results closer to the phantom. Table 2 represents the obtained numerical results. Visually, the results for VDA seem to be of better quality compared to the results for CTA. In particular, star rays are better distinguishable in the central part of the image and seem to have 


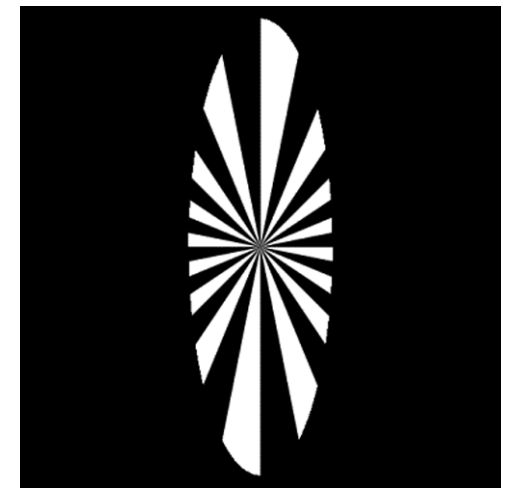

(a)

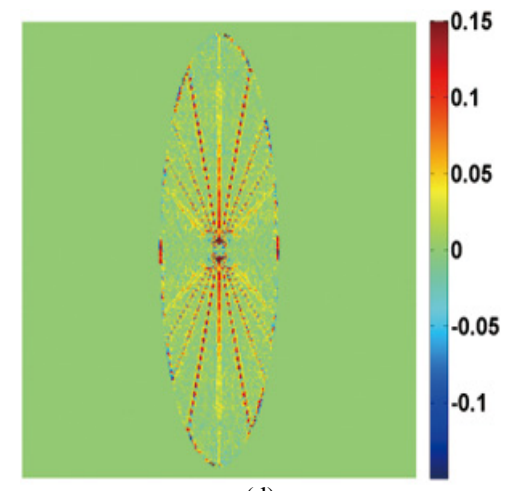

(d)

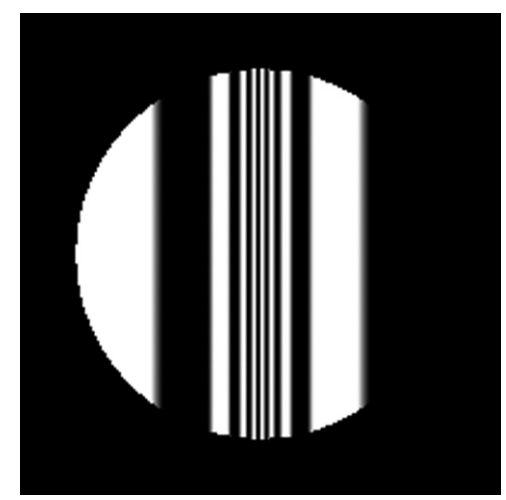

(g)

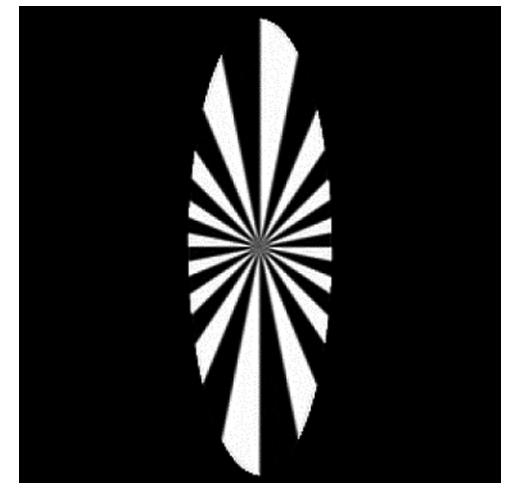

(b)

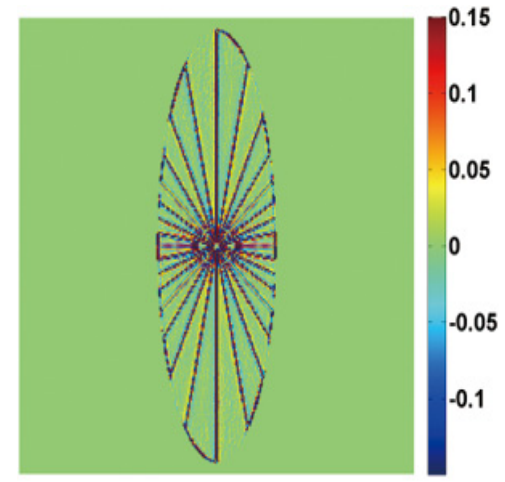

(e)

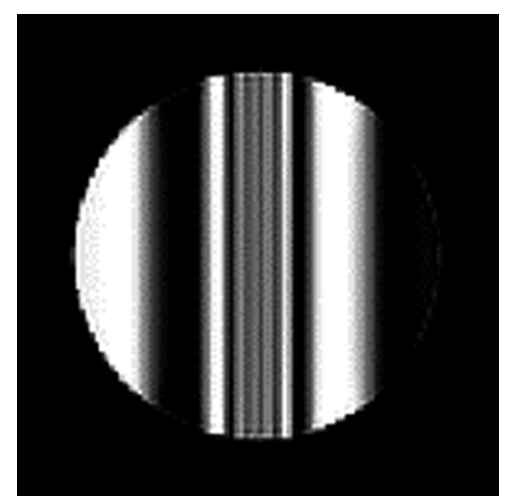

(h)

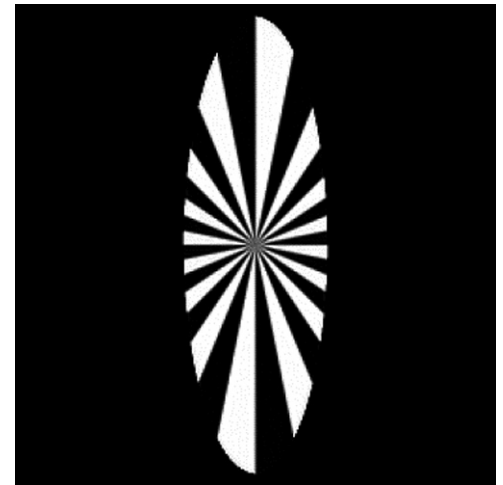

(c)

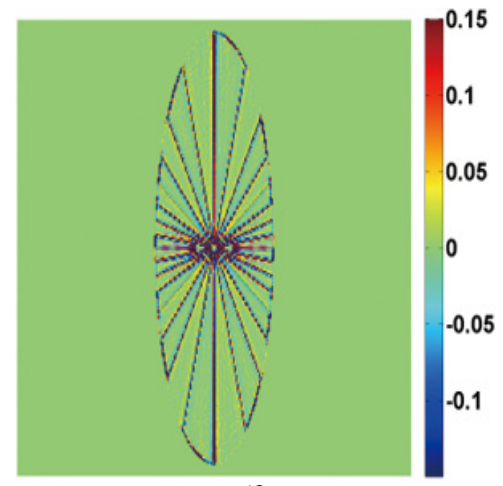

(f)

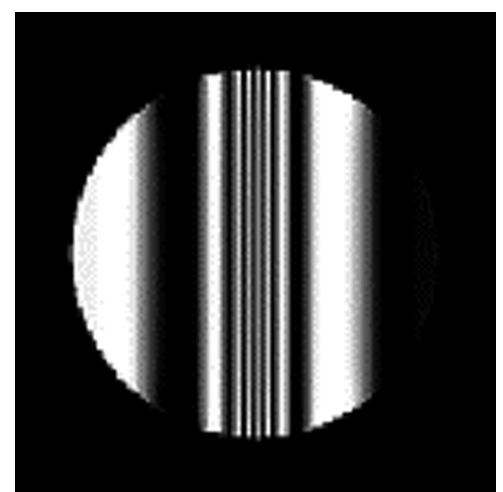

(i)

Fig. 5. Cross sections of Phantom 4 (a) and its CTA (b) and VDA (c) reconstructions by the plane $z=0.5$, corresponding error images (e, f), a difference image (d) as defined by Eq. (7), and magnified cross sections of Phantom 4 (g), its CTA (h) and VDA (i) reconstructions by the plane $x=-6.5$. (Colours are visible in the online version of the article; http://dx.doi.org/ 10.3233/XST-130410)

better vertical borders in the VDA reconstruction than in the CTA reconstruction (Fig. 5) of Phantom 4. For Phantom 5, inclusions near the tips of the ellipsoid are better distinguishable in the VDA reconstruction (Fig. 6). Interior of the phantom looks more uniform on the VDA reconstruction (Fig. 6(c)) than on the CTA reconstruction (Fig. 6(b)), whose artefacts might be confused with actual object features. The difference image (Fig. 6(d)) confirms these observations. Numerical results in Table 2 show that in terms of MSE VDA clearly outperforms CTA on both phantoms. The presented results suggest that VDA can 


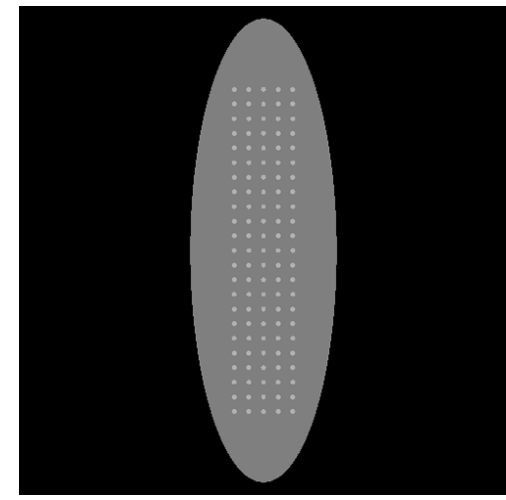

(a)
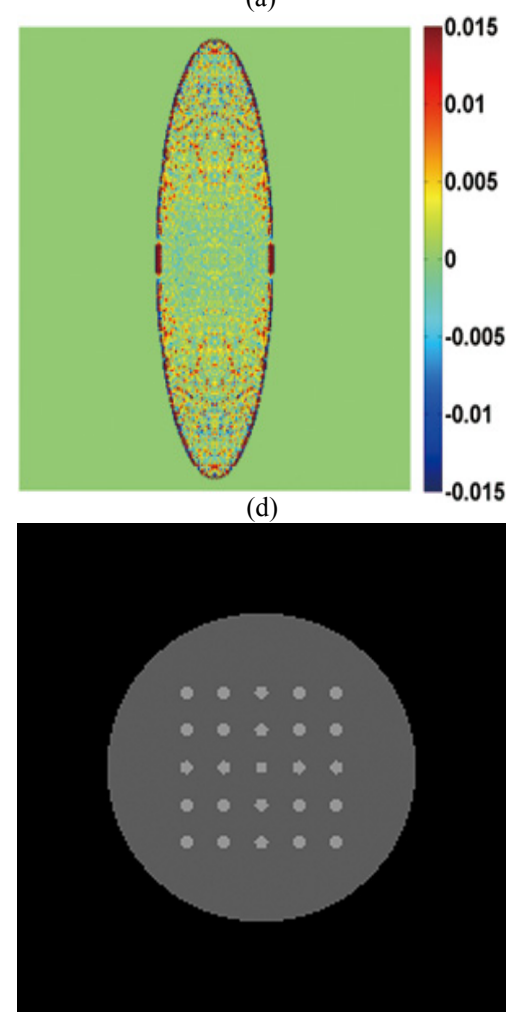

(g)

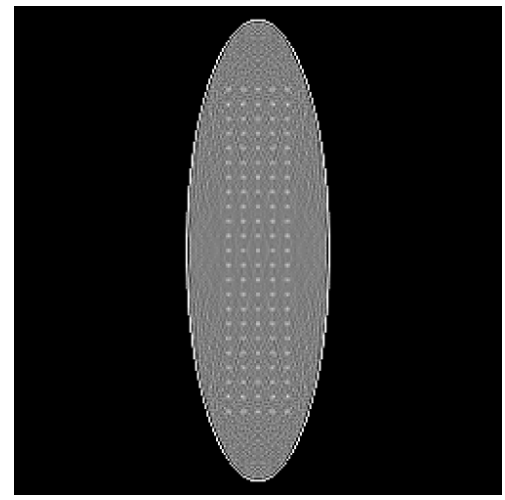

(b)
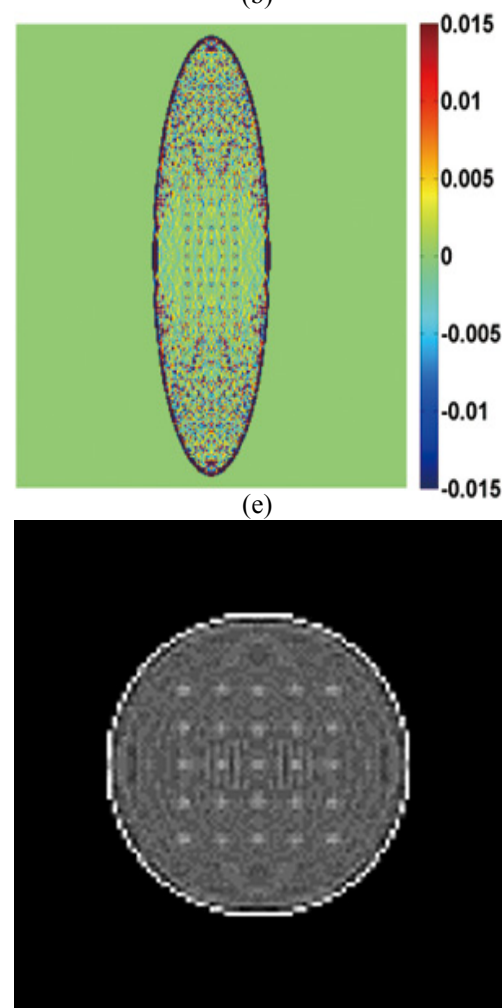

(h)

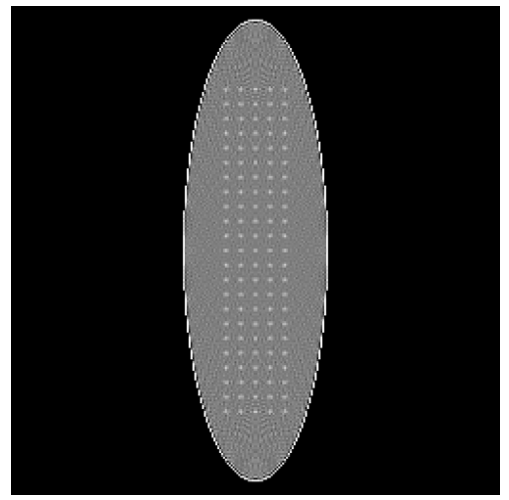

(c)
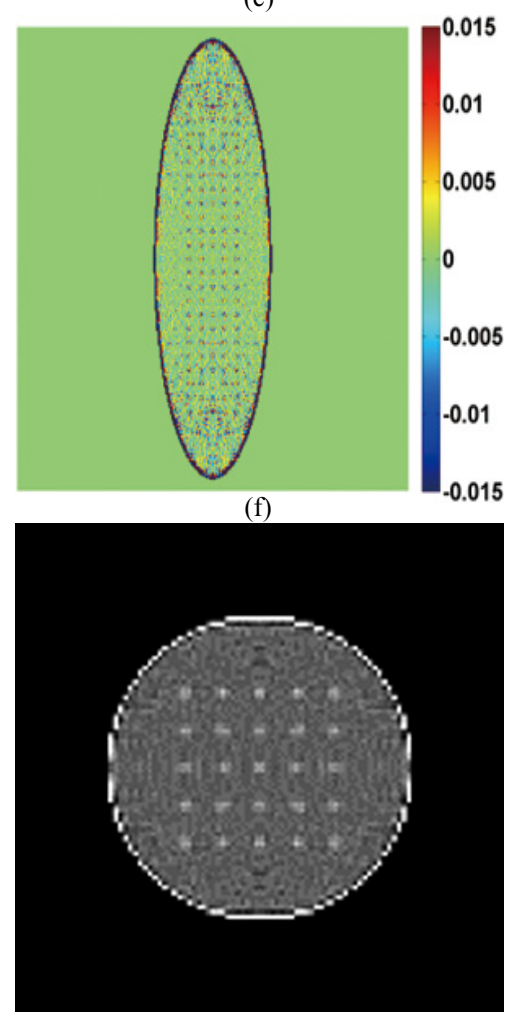

(i)

Fig. 6. Cross sections of Phantom 5 (a) and its CTA (b) and VDA (c) reconstructions by the plane $z=0.5$, corresponding error images (e, f), a difference image (d) as defined by Eq. (7), and magnified cross sections of Phantom 5 (g), its CTA (h) and VDA (i) reconstructions by the plane $x=-137.5((\mathrm{a}-\mathrm{c})$ and $(\mathrm{g}-\mathrm{i})$ windowed to $[0.45,0.55]$ for better visual contrast). (Colours are visible in the online version of the article; http://dx.doi.org/10.3233/XST-130410)

provide an ability to better handle small features in the objects than CTA.

\subsection{Simulations with noise}

In order to evaluate the proposed approach in more realistic situations the experiments shown in Sec- 
Table 2

MSE of the reconstructions of Phantoms 4-5 (shown in Fig. 4)

\begin{tabular}{ccc}
\hline & CTA & VDA \\
\hline Phantom 4 & & \\
$m=200$ & $2.27 \times 10^{-2}$ & $1.99 \times 10^{-2}$ \\
$m=500$ & $1.97 \times 10^{-2}$ & $1.66 \times 10^{-2}$ \\
Phantom 5 & & \\
$m=200$ & $2.82 \times 10^{-3}$ & $2.38 \times 10^{-3}$ \\
$m=500$ & $2.77 \times 10^{-3}$ & $2.29 \times 10^{-3}$ \\
\hline
\end{tabular}

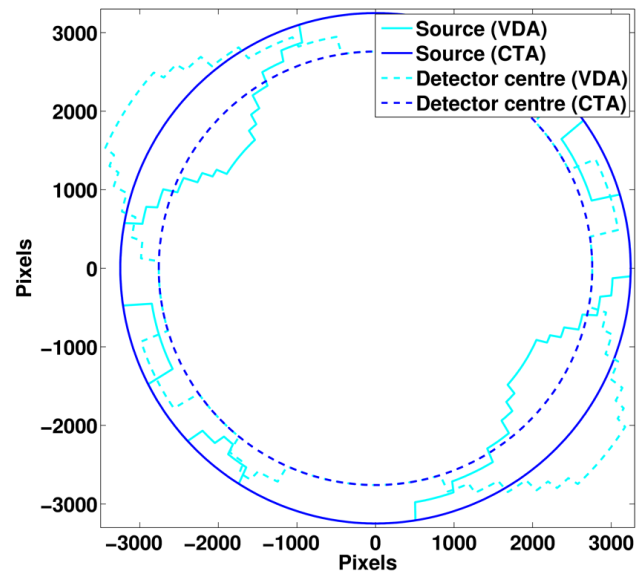

Fig. 7. Trajectories used for reconstruction of the pencil. (Colours are visible in the online version of the article; http://dx.doi.org/ 10.3233/XST-130410)

tions 3.1 and 3.2 were extended with noise simulations as follows. Consider a monochromatic X-ray tube which emits $N_{s}$ photons towards a detector element with the area of 1 square unit placed perpendicularly to the beam (to the line connecting the point X-ray source and the centre of the element) at the distance of $d_{s}$ from the source. Then, the average number of photons reaching the detector element $E$ at the distance of $d$ is

$$
N=N_{0} e^{-\int \mu(\xi) \mathrm{d} \xi}=N_{0} e^{-A}=N_{0} e^{-k \int g(\xi) \mathrm{d} \xi}=\frac{N_{s} d_{S}^{2} \cos \alpha}{d^{2}} e^{-k \int g(\xi) \mathrm{d} \xi},
$$

where $A$ is the ray integral calculated for the detector element $E$ with no noise introduced, $g(\xi)$ is the grey level of the phantom in the point $\xi, k$ is the scaling coefficient which matches $g(\xi)$ with the attenuation coefficient $\mu(\xi)$ of the object ( $k$ is assumed to be 1/100 in our simulations), $N_{0}$ is the number of photons emitted towards the considered detector element of the area of $S$ with $\alpha$ being the angle between the normal of the element and the beam (scattered photons were ignored). Then, the actual number of photons $N^{\prime}$ counted by $E$ can be selected according to Poisson statistics [7]. The noisy ray integral for the element $E$ can be calculated by

$$
A^{\prime}=-\ln \frac{N^{\prime}}{N_{0}}
$$

For each phantom from Sections 3.1 and 3.2, Eqs (8) and (9) were applied to the noiseless projections to obtain $K=10$ noisy sets of projection data (values $N_{s}=10^{5}$ and $N_{s}=10^{6}$ were used to represent 
Table 3

MSE of the noisy reconstructions of Phantoms 1-5, as described in Section 3.3

\begin{tabular}{|c|c|c|}
\hline & CTA & VDA \\
\hline \multicolumn{3}{|c|}{ Phantom $1, N_{s}=10^{5}$} \\
\hline$m=30$ & $1.07 \times 10^{-1}$ & $8.04 \times 10^{-2}$ \\
\hline$m=200$ & $1.88 \times 10^{-2}$ & $1.86 \times 10^{-2}$ \\
\hline \multicolumn{3}{|c|}{ Phantom $1, N_{s}=10^{6}$} \\
\hline$m=30$ & $9.26 \times 10^{-2}$ & $7.33 \times 10^{-2}$ \\
\hline$m=200$ & $1.51 \times 10^{-2}$ & $1.42 \times 10^{-2}$ \\
\hline \multicolumn{3}{|c|}{ Phantom 2, $N_{s}=10^{5}$} \\
\hline$m=30$ & $8.06 \times 10^{-2}$ & $8.00 \times 10^{-2}$ \\
\hline$m=200$ & $1.84 \times 10^{-2}$ & $1.73 \times 10^{-2}$ \\
\hline \multicolumn{3}{|c|}{ Phantom 2. $N_{s}=10^{6}$} \\
\hline$m=30$ & $7.71 \times 10^{-2}$ & $7.53 \times 10^{-2}$ \\
\hline$m=200$ & $1.39 \times 10^{-2}$ & $1.35 \times 10^{-2}$ \\
\hline \multicolumn{3}{|c|}{ Phantom $3, N_{s}=10^{5}$} \\
\hline$m=30$ & $6.34 \times 10^{-3}$ & $6.28 \times 10^{-2}$ \\
\hline$m=200$ & $4.82 \times 10^{-3}$ & $4.07 \times 10^{-3}$ \\
\hline \multicolumn{3}{|c|}{ Phantom $3, N_{s}=10^{6}$} \\
\hline$m=30$ & $2.16 \times 10^{-3}$ & $1.96 \times 10^{-3}$ \\
\hline$m=200$ & $8.05 \times 10^{-4}$ & $7.33 \times 10^{-4}$ \\
\hline \multicolumn{3}{|c|}{ Phantom $4 N=10^{5}$} \\
\hline$m=200$ & $2.40 \times 10^{-2}$ & $2.07 \times 10^{-2}$ \\
\hline$m=500$ & $2.02 \times 10^{-2}$ & $1.70 \times 10^{-2}$ \\
\hline \multicolumn{3}{|c|}{ Phantom $4 N_{s}=10^{6}$} \\
\hline$m=200$ & $2.28 \times 10^{-2}$ & $2.00 \times 10^{-2}$ \\
\hline$m=500$ & $1.98 \times 10^{-2}$ & $1.66 \times 10^{-2}$ \\
\hline \multicolumn{3}{|c|}{ Phantom 5, $N_{s}=10^{5}$} \\
\hline$m=200$ & $4.05 \times 10^{-3}$ & $3.25 \times 10^{-3}$ \\
\hline$m=500$ & $3.27 \times 10^{-3}$ & $2.64 \times 10^{-3}$ \\
\hline \multicolumn{3}{|c|}{ Phantom 5, $N_{s}=10^{6}$} \\
\hline$m=200$ & $2.95 \times 10^{-3}$ & $2.47 \times 10^{-3}$ \\
\hline$m=500$ & $2.82 \times 10^{-3}$ & $2.32 \times 10^{-3}$ \\
\hline
\end{tabular}

different noise levels and $d_{s}$ was equal to the source-object distance used in CTA in all simulations of this section). For each noisy projection dataset the reconstructions were built as described earlier and the mean values of $\operatorname{MSE}(\tilde{I}, I)$ over these $K$ reconstructions were gathered into Table 3, from which we see that VDA can yield better results in the presence of noise than CTA. For none of these cases, the latter outperforms VDA numerically, yielding reconstructions with visually similar or lower quality as it was already described in Section 3.2 in noiseless simulations.

\subsection{Real experiment}

To mimic a tomographic system with variable source and detector position, the following experiment was conducted using a desktop micro-CT system SkyScan-1172 (Bruker-MicroCT, Belgium). A piece of a pencil with a diameter of $7 \mathrm{~mm}$ and a length of $15 \mathrm{~mm}$ was used as an elongated object. For this object, seven full-angle datasets were obtained, each containing 600 images of $880 \times 666$ pixels, with the source-object distances ranging from 80.77 to $117.01 \mathrm{~mm}$. The source-detector distance was $216.392 \mathrm{~mm}$. A dataset obtained from the biggest distance was used during the reconstruction with CTA. Based on the CTA reconstruction, an approximate convex hull for VDA was created. In VDA for each projection angle the closest possible source position was calculated for this convex hull according to 


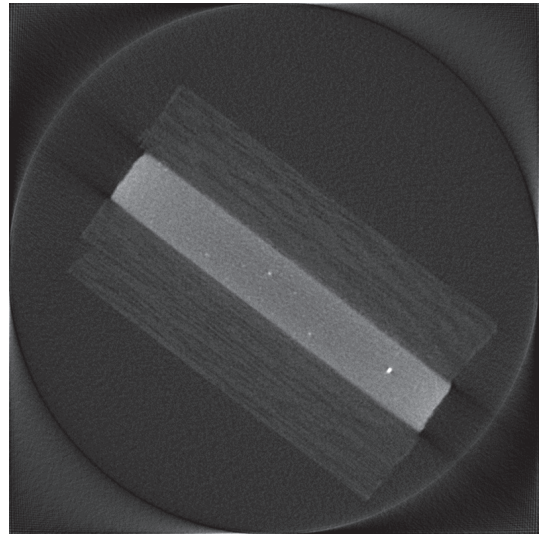

(a)

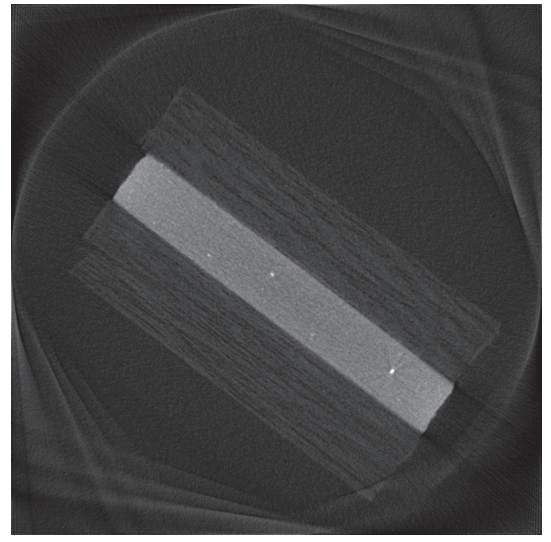

(b)

Fig. 8. Reconstructions of the central (containing optical axis) slice of the pencil with CTA (a) and VDA (b).

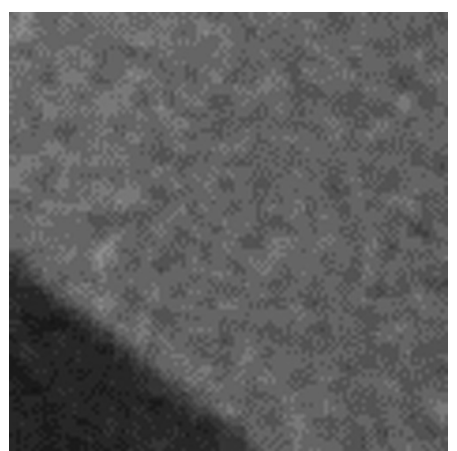

(a)

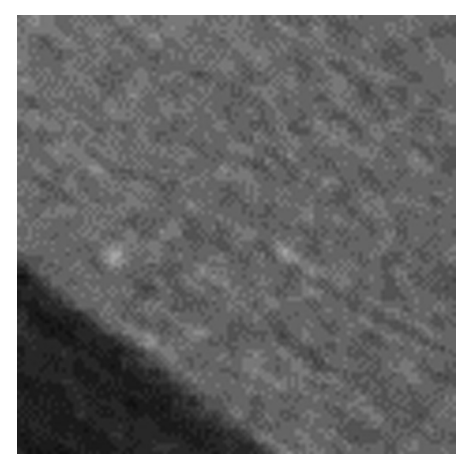

(b)

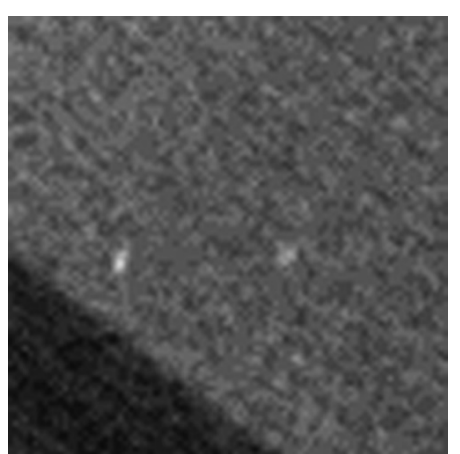

(c)

Fig. 9. Comparison of the CTA and VDA reconstructions of the pencil. Enlarged fragments of the CTA (a) and VDA (b) reconstructions of the central slice (Fig. 8), and the same region reconstructed with NRecon software (Bruker-MicroCT) (voxel size $13.4 \mu \mathrm{m})(\mathrm{c})$.

Eq. (5) and a projection was chosen from the dataset obtained from the smallest distance bigger than or equal to the distance from the calculated source position to the centre of rotation. Resulting trajectories are presented in Fig. 7.

Both the CTA and VDA reconstructions were performed on the $880 \times 880 \times 666$ voxels reconstruction grid with a voxel size of $19.4 \mu \mathrm{m}$ using 700 iterations of SIRT. Figure 8 presents the reconstructions. Visually, both reconstructions seem to have comparable quality. However, in the enlarged portions of the reconstructions shown in Figs 9(a) and 9(b) a border between wood and graphite seems to have better contrast in the VDA reconstruction and two dense particles in the middle of the image are easier visually distinguishable. Figure 9(c) presents the reconstruction of the same region obtained with NRecon software (Bruker-MicroCT) from the dataset with the smallest source-object distance, the voxel size is $13.4 \mu \mathrm{m}$. As the region lies far from the edge of the field-of-view, artefacts caused by the truncated projections are negligible in this part of the NRecon reconstruction. We therefore consider the NRecon reconstruction as the ground truth for this region. This reconstruction shows that the above mentioned differences in the CTA and VDA reconstructions are not the artefacts of the latter, but rather the features truly presented in the object. Hence, experimental studies agree with the simulations described in Section 3.2, showing the ability of VDA to produce reconstructions which are superior to those produced 
by CTA in the realistic setup.

\section{Discussion}

The proposed approach allows to exploit prior knowledge of the object's shape and size to optimize the detector usage and to obtain more detailed information when scanning an elongated object, increasing the reconstruction quality. An approximate convex hull of the object can be created from a preparatory scan (in clinical CT), a series of pictures of the object in optical range (in micro-CT) or a CAD model (in industrial CT). Implementation of the source position selection algorithm is straightforward and easily adaptable to various setups, e.g. systems with constant object-detector distance (rather than a system with constant source-detector distance, considered in the paper). The data collected can be immediately reconstructed with an algebraic reconstruction procedure, while analytical methods require rebinning, possibly leading to loss of quality.

Possible applications of the proposed approach include mobile tomographical devices for use in the field and tomography of objects that have substantial differences in all three dimensions, such as electronic components. Currently these objects are imaged in helical or cone-beam stacked mode and the source-object distance is defined by the second biggest dimension, no matter how small the third one is. Use of the variable distance approach will allow to better exploit the dimension differences in this case.

\section{Conclusion}

We proposed the variable distance approach for fan- and cone-beam CT scanning. This approach is based on the modification of the classic circular trajectory according to prior information about the object's convex hull which is used to take projections from as small as possible distances to the object for every projection angle providing that the truncation is avoided. Our experiments showed that the proposed approach can lead to more accurate reconstructions with lower errors. Reconstruction of the real dataset demonstrated an ability of the approach to reveal more details in the object compared to the conventional circular trajectory.

\section{Acknowledgments}

The authors would like to thank A. Sasov from Bruker-MicroCT and Elke Van de Casteele for their help in the data acquisition for the real experiment. This work was financially supported by the BOF LP project 25778 and iMinds (Interdisciplinary Institute for Technology, a research institute founded by the Flemish Government). K.J.B. was supported by the Netherlands Organisation for Scientific Research (NWO), programme 639.072.005.

\section{References}

[1] S. Abbas, J. Min and S. Cho, Super-sparsely view-sampled cone-beam CT by incorporating prior data, Journal of X-Ray Science and Technology 21(1) (2013), 71-83.

[2] K.J. Batenburg and J. Sijbers, DART: A practical reconstruction algorithm for discrete tomography, IEEE Transactions on Image Processing 20(9) (Sep 2011), 2542-2553. 
[3] R.L. Eisner, W.A. Fajman, D.J. Nowak and R.I. Pettigrew, Improved image quality with elliptical orbits and distanceweighted backprojection SPECT reconstruction, Annals of Nuclear Medicine 2 (1988), 107-110.

[4] M. Fedrigo, A. Wenger and C. Hoeschen, Investigating tomographic reconstruction with a priori geometrical information, Journal of X-Ray Science and Technology 20(1) (2012), 1-10.

[5] S.C. Gottschalk, D. Salem, C.B. Lim and R.H. Wake, SPECT resolution and uniformity improvements by noncircular orbit, Journal of Nuclear Medicine 24(9) (1983), 822-828.

[6] J. Gregor and T. Benson, Computational analysis and improvement of SIRT, IEEE Transactions on Medical Imaging, 27(7) (Jul 2008), 918-924.

[7] H.Q. Guan and R. Gordon, Computed tomography using algebraic reconstruction techniques (ARTs) with different projection access schemes: A comparison study under practical situations, Physics in Medicine and Biology 41(9) (Sep 1996), 1727-1743.

[8] P.M. Joseph, An improved algorithm for reprojecting rays through pixel images, IEEE Transactions on Medical Imaging 1(3) (1982), 192-196.

[9] A. Laurentini, The visual hull concept for silhouette-based image understanding, IEEE Transactions on Pattern Analysis and Machine Intelligence 16(2) (Feb 1994), 150-162.

[10] W.J. Palenstijn, K.J. Batenburg and J. Sijbers, The ASTRA tomography toolbox, in: 13th International Conference on Computational and Mathematical Methods in Science and Engineering, CMMSE 2013, 2013.

[11] T.S. Pan, D.S. Luo, V. Kohli and M.A. King, Influence of OSEM, elliptical orbits and background activity on SPECT 3D resolution recovery, Physics in Medicine and Biology 42(12) (Dec 1997), 2517-2529.

[12] E.Y. Sidky, C.M. Kao and X. Pan, Accurate image reconstruction from few-views and limited-angle data in divergentbeam CT, Journal of X-Ray Science and Technology 14(2) (2006), 119-139.

[13] S. Tang, Y. Yang and X. Tang, Practical interior tomography with radial Hilbert filtering and a priori knowledge in a small round area, Journal of X-Ray Science and Technology 20(4) (2012), 405-422.

[14] A. Todd-Pokropek, Non-circular orbits for the reduction of uniformity artefacts in SPECT, Physics in Medicine and Biology 28(3) (1983), 309-313.

[15] D. Xia, S. Cho, J. Bian, E.Y. Sidky, C.A. Pelizzari and X. Pan, Tomosynthesis with source positions distributed over a surface, in: Proceedings of the SPIE - The International Society for Optical Engineering, SPIE - The International Society for Optical Engineering. Medical Imaging 2008: Physics of Medical Imaging, San Diego, CA, USA, 18-21 Feb 2008, pp. 69132A-1-7. 